\title{
On the discount sum of Bernoulli random variables
}

\author{
Ka-Sing Lau*, Alan Ho \\ Deparment of Mathenatics, University of Pitshurgh. Pittiburgh PA 15260). (S4 \\ Received 7 February 1995; received in revised form 11 April 1996
}

Dedicated to Professor C.R. Rao on the occasion of his seventy-fifth birahda!

\begin{abstract}
Let $\left\{\boldsymbol{\varepsilon}_{n}\right\}_{n=1}^{x}$ be i.i.d. Bernoulli random variables. For $\frac{1}{2}<\rho<1$, let $\boldsymbol{X}=\sum_{n}^{x} \rho^{\prime \prime} \varepsilon_{n}$ be the discount sum and let $\mu_{p}$ be the distribution measure. It is known that if $\rho{ }^{1}$ is a P.V. number. then $\mu_{p}$ is continuously singular. In this paper we use a Markov chain technique to obtain the precise $L^{2}$-dimension of such measures. In particular for $\rho=(\sqrt{5}-1) / 2$, we use a device of Strichartz et al and the renewal equation to derive a formula for the $L^{\prime \prime}$-dimension and the entropy dimension of the corresponding $\mu_{\mu}$. (C) 1997 Elsevier Science B.V.
\end{abstract}

\section{Introduction}

Let $\left\{\varepsilon_{n}\right\}_{n=1}^{\infty}$ be i.i.d. Bernoulli random variables, i.e., $\varepsilon_{n}$ takes values 0 and 1 with probability $\frac{1}{2}$ each. For $0<\rho<1$, let $X=\sum_{k=1}^{\infty} \rho^{k} \varepsilon_{k}$ be the geometric discount sum of the $\varepsilon_{n}$ 's, and let $\mu_{\rho}$ be the corresponding distribution measure. $\mu_{\rho}$ is the convolution of the sequence $\left\{\frac{1}{2}\left(\delta_{0}+\delta_{\beta^{k}}\right)\right\}_{k=1}^{\infty}$ of point mass measures and is called an infinitely convolved Bernoulli measure (ICBM). Such measures possess very intricate and interesting properties concerning their absolute continuity and singularity. The first important work on this was due to Jessen and Wintner in 1935. They showed that $\mu_{\rho}$ is always a continuous measure, and is either purely singular or purely absolutely continuous. For $0<\rho<\frac{1}{2}, \mu_{\rho}$ is singular and is supported by a Cantor-type set on $[0, \rho /(1-\rho)]$, which has Hausdorff dimension $|\ln 2 / \ln \rho|$. For $\rho=\frac{1}{2}, X$ is a binary number with random digits 0,1 that occur with probability $\frac{1}{2}$. It is clear that $\mu_{1 / 2}$ is a uniform distribution on $[0,1]$. Furthermore, it is easy to show that if $\rho=(1 / 2)^{1 \cdot n}$, for $n=1,2 \ldots$, then $\mu_{p}$ is essentially an $n$-fold convolution of $\mu_{1,2}$ and is hence also absolutely continucus. In view of these examples of increasing smoothness of $\mu_{\mu}$, there was a naive conjecture that for $\frac{1}{2}<\rho<1, \mu_{\rho}$ is absolutely continuous. This was disproved by Erdös (1939) by observing that the regularity of $\mu_{p}$, depends on the algebraic properties of $\rho$. Recall

\footnotetext{
* Corresponding author. E-mail: lauks+ (upitt.edu.
} 
that $\beta$ is called an algebraic integer if it is a root of a polynomial $L$ with integer coefficients and leading coefficient 1; assuming that $L$ is of smallest degree, we call $\beta^{\prime}$ an algebraic conjugate of $\beta$ if $\beta^{\prime}$ is another root of $L$. The following are two fascinating results in this direction. The first one is due to Erdös (1939) and Salem (1962), the second one is due to Garsia (1962): Suppose $\frac{1}{2}<\rho<1$ and $\beta=\rho^{-1}$ is an algebraic integer with algebraic conjugates $\beta_{1}, \ldots, \beta_{s}$. Then

(i) $\beta$ is a Pisot-Vijayaraghavan (P.V.) number (i.e. $|\beta|>1$ and $\left|\beta_{i}\right|<1, i=1, \ldots, s$ ) if and only if $\hat{\mu}_{\rho}(\xi) \nrightarrow 0$ as $|\xi| \rightarrow \infty$ where $\hat{\mu}_{\rho}$ is the Fourier transformation of $\mu_{\rho}$. In particular, $\mu_{\rho}$ is singular by the Riemann Lebesgue lemma.

(ii) If $\beta \prod_{\left|\beta_{i}\right|>1} \beta_{i}=2$, then $\mu_{\rho}$ is absolutely continuous. Note that the assumption actually implies that $\left|\beta_{i}\right|$ is greater than 1 for all $i=1, \ldots, s$ (see (Garsia, 1962 Lemma 4.7)), in contrast to case (i).

The basic idea is that for each $n$, the values of the sum $\sum_{k=1}^{n} \rho^{k} \varepsilon_{k}$ in case (i) repeat very often so that $\mu_{\rho}$ is singular, and in case (ii) the values do not repeat and are more or less equally spaced so that $\mu_{\rho}$ is absolutely continuous. In another direction, Erdös (1940) showed that

(iii) There exists $\rho_{0}$ sufficiently close to 1 such that for almost all $\rho_{0}<\rho<1, \mu_{\rho}$ is absolutely continuous.

He also made the conjecture that $\mu_{\rho}$ is absolutely continuous for almost all $\frac{1}{2}<\rho<1$. There was no progress on this problem for a long time. Recently interest has been re-kindled, however, due to the development of the geometry of fractals, dynamical systems and iterated function systems (cf. Alexander and Yorke (1984), Bovier, Falconer (1990), Hu (to appear), Lalley, Lau and Ngai (to appear) and the references there). Let

$$
S_{j}(x)=\rho_{j} x+b_{j}, \quad x \in \mathbb{R}^{d}
$$

where $0<\rho_{j}<1$ and $b_{j} \in \mathbb{R}^{d}, j=1, \ldots, m$, be similitudes and let $\left\{a_{j}\right\}_{j=1}^{m}$ be associated weights for the $\left\{S_{j}\right\}_{j=1}^{m}$. Then there exists a unique compact subset $K$ and a unique probability measure $\mu$ satisfying

$$
K=\bigcup_{j=1}^{m} S_{j}(K) \text { and } \quad \mu=\sum_{j=1}^{m} a_{j} \mu \circ S_{j}^{-1} .
$$

$K$ is called a self-similar set and $\mu$ is called a self-similar measure (Falconer, 1990; Hutchinson, 1981). Both $K$ and $\mu$ can be obtained through iteration of $\left\{S_{j}\right\}_{j=1}^{m}$, starting from any compact subset $E$ in $\mathbb{R}^{d}$ and any probability measure on $E$. For the case

$$
S_{1}(x)=\rho x, \quad S_{2}(x)=\rho x+(1-\rho), \quad x \in \mathbb{R}
$$

with weights $\frac{1}{2}$ each, the self-similar measure is actually the ICBM $\mu_{\rho}$, modulo a scaling factor, because

$$
\hat{\mu}(\xi)=\mathrm{e}^{\mathrm{i} \xi / 2} \prod_{n=1}^{\infty} \cos \left(\frac{1-\rho}{2} \rho^{n} \xi\right) \quad \text { and } \quad \hat{\mu}_{\rho}(\xi)=\mathrm{e}^{\mathrm{i} \xi / 2(1-\rho)} \prod_{n=1}^{\infty} \cos \left(\frac{\rho^{n}}{2} \xi\right) .
$$


If $0<\rho<\frac{1}{2}, K$ is a Cantor-type set and is the disjoint union of the two copies $S_{1}\left(K_{2}\right)$ and $S_{2}(K)$. This nonoverlapping property of the components has been extended to the so-called open set condition (Hutchinson, 1981), and is one of the most fundamental concepts in the geometry of fractals. On the other hand, very few results are known without such a condition. The ICBM $\mu_{f}$, with $\frac{1}{2}<\rho<1$ is the simplest important example of the overlapping case $\left(S_{0}[0,1] \cap S_{1}[0,1] \neq \emptyset\right)$ and can be used as a pilot case to understand the more general situation (Lau and Ngai, to appear).

For the recent developments, Solomyak (1995) solved Erdös' conjecture following (iii). When $\mu_{p}$ is singular there are a number of ways to interpret the exponent $x$ when $\mu(x-h x+h) \sim c h^{x}$ as $h \rightarrow 0$. For example, locally $x$ can be regarded as tine the so-called local dimension of $\mu$ at $x$, while globally, $x$ is related to the Hausctorff dimension, entropy dimension, $L^{p}$-dimension, etc. The entropy dimension of $\mu_{,}$, when $\rho^{\prime}$ is a P.V. number was studied by Garsia (1963) and has been re-examinated (in particular when $\rho^{-1}=(\sqrt{5}+1) / 2$, the golden number) by Alexander and Yorke (1984), Alexander and Zagier (1991), Ledrappier and Porzio (preprint) and Prsytycki and Unbanski (1989). The $L^{2}$-dimension of such measures has also been calculated by Lau (1991, 1993). In Lau (1991) the open set condition of the iterated function system is extended to a weaker separation condition which includes the ase that $\mu$ is a P.V. number.

In this paper we discuss two different ways to calculate the dimensions of the singular ICBM. We first use the Markov chain approach to obtain the exact $L^{2}$-dimension of the ICBM when $\rho^{-1}$ is a P.V. number (Theorem 3.7). This simplifies the indirect approach in Lau (1993). For $\rho=(\sqrt{5}-1) 2$. Strichartz et al. (to appear) have an innovative idea of replacing the two maps in (1.2) by three maps that satisfy the open set condition, which yields via the self-similar identity (1.1) a complementary set of identities (see Eq. (4.2)). By using this and the renewal equation, we obtain a formula of the $L^{\prime \prime}$-dimension and the entropy dimension for such $\mu_{\mu}$ (Theorem 4.1).

We organize the paper as follows. In Section 2 we review some definitions of the dimensions of a measure and reformulate them into the setting we need. In Section 3 we consider the $L^{2}$-dimension of the ICBM generated by the P.V. numbers and in Section 4 we describe the $L^{\prime \prime}$-dimension formula of the ICBM corresponding to the golden number. The technical details concerning Section 4 are proved in Lau and $\mathrm{N} g a \mathrm{a}$ (to appear). In Section 5 we discuss some open problems and remarks. We also include several graphs that approximate the density of the measures.

\section{Preliminaries}

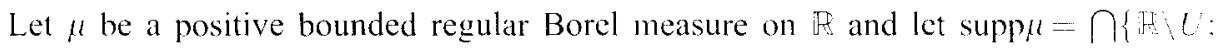
$U$ is open and $\mu(U)=0\}$ be the support of $\mu$. For convenience we assume that $\mu$ has a bounded support. If $\mu$ is absolutely continuous then the density of $\mu$ is $\lim /, \quad$. $\mu\left(B_{h}(x)\right) 2 h$ for almost all $x$ (with respect to Lebesgue measure), where $B_{h}(x)$ denotes the ball of radius $h$ centered at $x$ in this case $\mu\left(B_{h}(x)\right) \sim c h$ as $h \rightarrow 0$. We will 
define some notions of fractional density and dimension to handle the case where $\mu\left(B_{h}(x)\right) \sim c h^{\alpha}, 0 \leqslant \alpha \leqslant 1$.

For $h>0$, let $\left\{x_{i}=i h: i \in \mathbb{Z}\right\}$ be a lattice of $\mathbb{R}$ and let $E_{i}(h)\left(=E_{i}\right)=\left(x_{i-1}, x_{i}\right]$. For $1 \leqslant p<\infty$, let

$$
S^{p}(h)=\sum_{i} \mu\left(E_{i}(h)\right)^{p}
$$

be the $p$-variation of $\mu$. It is clear that when $p=1, S^{\mathrm{l}}(h)=\|\mu\|$ is the total variation of $\mu$. We define the $L^{p}$-dimension of $\mu$ as follows: For $1<p<\infty$,

$$
\operatorname{dim}_{p}(\mu)=\lim _{h \rightarrow 0^{+}} \frac{\ln S^{p}(h)}{(p-1) \ln h}
$$

and for $p=1$,

$$
\operatorname{dim}_{1}(\mu)=\lim _{h \rightarrow 0^{+}} \frac{\sum_{i}\left\{\mu\left(E_{i}\right) \ln \mu\left(E_{i}\right): E_{i} \cap \operatorname{supp} \mu \neq \emptyset\right\}}{\|\mu\| \ln h} .
$$

Also we let $\operatorname{dim}_{\infty}(\mu)=\lim _{h \rightarrow 0^{+}} \ln \sup \mu\left(E_{i}\right) / \ln h$. Note that $\operatorname{dim}_{1}(\mu)$ is also called the entropy dimension (Rényi, 1957), $\operatorname{dim}_{2}(\mu)$ the correlation dimension, and $\operatorname{dim}_{p}(\mu)$, $p>1$ the generalized Rényi dimension. Heuristically, $\operatorname{dim}_{1}(\mu)$ is the limit of $\operatorname{dim}_{p}(\mu)$ as $p \rightarrow 1$ by L'Hôspital's rule. We can also define $\underline{\operatorname{dim}}_{p}(\mu), \overline{\operatorname{dim}}_{p}(\mu)$ by replacing the lim with limsup and liminf. It is straightforward to check that for $1<p<\infty$,

$$
\begin{aligned}
& {\operatorname{dim}_{p}}_{p}(\mu)=\sup \left\{\frac{\alpha}{p-1}: \varlimsup_{h \rightarrow 0^{+}} \frac{S^{p}(h)}{h^{\alpha}}<\infty\right\}, \\
& \overline{\operatorname{dim}}_{p}(\mu)=\inf \left\{\frac{\alpha}{p-1}: 0<\varliminf_{h \rightarrow 0^{+}} \frac{S^{p}(h)}{h^{\alpha}}\right\} ;
\end{aligned}
$$

and $\operatorname{dim}_{p}(\mu)=\alpha /(p-1)$ if

$$
0<\varliminf_{h \rightarrow 0^{+}} \frac{S^{p}(h)}{h^{\alpha}} \leqslant \varlimsup_{h \rightarrow 0^{+}} \frac{S^{p}(h)}{h^{x}}<\infty .
$$

The converse of $(2.1)$ is not true in general. The $L^{P}$-dimension has a very useful alternative form in terms of the integral

$$
I^{p}(h)=\int_{-\infty}^{\infty} \mu\left(B_{h}(x)\right)^{p} \mathrm{~d} x
$$

Proposition 2.1. For $1<p<\infty$,

$$
\operatorname{dim}_{p}(\mu)=\lim _{h \rightarrow 0^{+}} \frac{1}{p-1}\left(\frac{\ln I^{p}(h)}{\ln h}-1\right),
$$

and for $p=1$,

$$
\operatorname{dim}_{1}(\mu)=\lim _{h \rightarrow 0^{+}}\left(\frac{\ln \int \mu\left(B_{h}(x)\right) \ln \mu\left(B_{h}(x)\right) \mathrm{d} x}{\|\mu\| \ln h}-1\right) .
$$


It is a direct consequence of the following lemma:

Lemma 2.2. For $1 \leqslant p<\infty, h>0$,

$$
S^{p}(h) \leqslant \frac{1}{h} I^{p}(h) \leqslant 4^{p} S^{p}(h) .
$$

Furthermore for $\mu(\mathbb{R}) \leqslant 1$ and for $:>0$, there exists $h_{0}$ such that for $0<h<h_{0}$.

$$
\begin{aligned}
4 \sum_{i} \mu\left(E_{i}(h)\right) \ln \mu\left(E_{i}(h)\right) & \leqslant \frac{1}{h} \int_{-\infty}^{\infty} \mu\left(B_{h}(x)\right) \ln \mu\left(B_{h}(x)\right) \mathrm{d} x \\
& \leqslant \sum_{i} \mu\left(E_{i}(h)\right) \ln \mu\left(E_{i}(h)\right)+i
\end{aligned}
$$

where the sums are taken over all the $E_{i}(h)$ hating nonempty intersection with supp 1.

Proof. We first observe that for $x_{i}=i h$.

$$
\mu\left(E_{i}(h)\right)=\frac{1}{h} \int_{E_{i}(h)} \mu\left(E_{i}(h)\right) \mathrm{d} x \leqslant \frac{1}{h} \int_{E_{i}(h)} \mu\left(B_{h}(x)\right) \mathrm{d} x \leqslant \mu\left(B_{2 h}\left(x_{i}\right)\right) .
$$

By Jensen's inequality with $\varphi(u)=|u|^{\prime \prime}$, we have

$$
S^{\prime}(h) \leqslant \sum_{i}\left(\int_{E,(h)} \mu\left(B_{h}(x)\right) \frac{\mathrm{d} x}{h}\right)^{p} \leqslant \sum_{i} \int_{b(h)} \mu\left(B_{h}(x)\right)^{\prime} \frac{\mathrm{d} x}{h}=\frac{1}{h} I^{\prime \prime}(h) .
$$

On the other hand,

$$
\frac{1}{h} I^{\prime \prime}(h)=\frac{1}{h} \sum_{i} \int_{E,(h)} \mu\left(B_{h}(x)\right)^{p} \mathrm{~d} x \leqslant \sum_{i} \mu\left(B_{2 h}\left(x_{i}\right)\right)^{p} \leqslant 4^{\prime \prime} S^{p}(h)
$$

and the first assertion follows. For the second assertion, we use Jensen's inequality with $\varphi(u)=u \ln u, u>0$ and the last inequality of (2.3) to obtain

$$
\sum_{i} \mu\left(B_{2 h}\left(x_{i}\right)\right) \ln \mu\left(B_{2 h}\left(x_{i}\right)\right) \leqslant \frac{1}{h} \int_{-x}^{x} \mu\left(B_{h}(x)\right) \ln \mu\left(B_{h}(x)\right) \mathrm{d} x .
$$

The left inequality now follows from $\varphi(u)+\varphi(v) \leqslant \varphi(u+v), u, v>0$. For the right inequality we make use of the fact that $\varphi(u)$ is negative and decreasing for $0<\|<\mathrm{e}$. if all the atoms $a$ of $\mu$ satisfy $\mu\{a\}<\mathrm{e}^{-1}$, then there exists $h_{0}$ such that for $0<h<h_{0}$. $\mu\left(E_{i}(h)\right)<\mathrm{e}^{-1}$ for all $i$, and hence

$$
\begin{aligned}
\frac{1}{h} \int_{-x}^{x} \mu\left(B_{h}(x)\right) \ln \mu\left(B_{h}(x)\right) \mathrm{d} x & =\frac{1}{h} \sum_{i} \int_{b} \mu\left(B_{h}(x)\right) \ln \mu\left(B_{h}(x)\right) \mathrm{d} x \\
& \leqslant \frac{1}{h} \sum_{i} \int_{b} \mu\left(E_{i}\right) \ln \mu\left(E_{i}\right) \mathrm{d} x=\sum_{i} \mu\left(E_{i}\right) \ln \mu\left(E_{i}\right) .
\end{aligned}
$$

If the measure contains atoms $a$ such that $\mu\{a\} \geqslant \mathrm{e}^{-1}$, then it can have at most two such $a$. We can calculate separately the intervals that contains these $a$ 's and adjust the inequality by the $c$. 


\section{3. $L^{2}$-dimension of ICBM}

For convenience we let $\boldsymbol{\varepsilon}_{0}=0$. Let $\boldsymbol{X}=\sum_{k=0}^{\infty} \rho^{n} \boldsymbol{\varepsilon}_{n}$ be the discount sum of the Bernoulli random variables and let $\mu_{\rho}$ be the corresponding distribution measure as in Section 1. With no confusion we will omit the subscript $\rho$ in $\mu_{\rho}$. Let $\boldsymbol{X}_{n}=\sum_{k=0}^{n} \rho^{k} \varepsilon_{k}$ and let $\mu_{n}$ be the corresponding distribution. Then $\mu_{n}$ is the $n$-th convolution of $\frac{1}{2}\left(\delta_{0}+\delta_{\rho^{k}}\right), 1 \leqslant k \leqslant n$,

$$
\mu_{n}\{x\}=\frac{1}{2^{n}} \#\left\{\sum_{k=0}^{n} \rho^{k} \varepsilon_{k}: \sum_{k=0}^{n} \rho^{k} \varepsilon_{k}=x, \varepsilon_{k}=0 \text { or } 1, k=0, \ldots, n\right\},
$$

and $\left\{\mu_{n}\right\}_{n=1}^{\infty}$ converges to $\mu$ in distribution.

Lemma 3.1. Let $\left\{\mu_{n}\right\}$ and $\mu$ be defined as above, then for $n>0$,

$$
\mu\left(B_{\rho^{\prime \prime}}(x)\right) \leqslant \mu_{n}\left(B_{\rho^{\prime \prime} /(1-\rho)}(x)\right) \leqslant \mu\left(B_{2 \rho^{\prime \prime} /(1-\rho)}(x)\right) .
$$

Proof. It suffices to show that

$$
\left\{\omega: \boldsymbol{X}(\omega) \in B_{\rho^{\prime \prime}}(x)\right\} \subseteq\left\{\omega: \boldsymbol{X}_{n}(\omega) \in B_{\rho^{\prime \prime} /(1-\rho)}(x)\right\} \subseteq\left\{\omega: \boldsymbol{X}(\omega) \in B_{2 \rho^{\prime \prime} /(1-\rho)}(x)\right\}
$$

Indeed if $\boldsymbol{X}(\omega) \in B_{\rho^{\prime \prime}}(x)$, then

$$
\left|\boldsymbol{X}_{n}(\omega)-x\right| \leqslant\left|\boldsymbol{X}_{n}(\omega)-\boldsymbol{X}(\omega)\right|+|\boldsymbol{X}(\omega)-x| \leqslant \frac{\rho^{n+1}}{1-\rho}+\rho^{n}=\frac{\rho^{n}}{1-\rho} .
$$

The second identity can be obtained by the same argument.

Using this lemma we can reduce the calculation of the $L^{p}$-dimension of $\mu$ to the discrete measures $\mu_{n}$.

Proposition 3.2. There exist $C_{1}, C_{2}>0$ such that for any $I C B M \mu$ and for any $n>0$,

$$
C_{1} \sum_{i} \mu_{n}\left(E_{i}\left(\rho^{n}\right)\right)^{p} \leqslant S^{p}\left(\rho^{n}\right) \leqslant C_{2} \sum_{i} \mu_{n}\left(E_{i}\left(\rho^{n}\right)\right)^{p}
$$

Consequently,

$$
\operatorname{dim}_{p}(\mu)=\lim _{n \rightarrow \infty} \frac{\ln \sum_{i} \mu_{n}\left(E_{i}\left(\rho^{n}\right)\right)}{n(p-1) \ln \rho}
$$

and

$$
\operatorname{dim}_{1}(\mu)=\lim _{n \rightarrow \infty} \frac{\sum_{i} \mu_{n}\left(E_{i}\left(\rho^{n}\right)\right) \ln \mu_{n}\left(E_{i}\left(\rho^{n}\right)\right)}{n \ln \rho} .
$$


Let $\tilde{\boldsymbol{\varepsilon}}_{0}=0$ and let $\left\{\tilde{\boldsymbol{\varepsilon}}_{n}\right\}_{n=1}^{\infty}$ be i.i.d. random variables taking values $-1,0.1$ with probability $\frac{1}{4}, \frac{1}{2}, \frac{1}{4}$ respectively. Let

$$
\boldsymbol{Z}_{n}=\sum_{k=-0}^{n} \rho^{k} \tilde{\boldsymbol{\varepsilon}}_{k}, \quad \boldsymbol{Z}=\sum_{k=0}^{\infty} \rho^{k} \tilde{\boldsymbol{\varepsilon}}_{k},
$$

and let $\gamma_{n}$ and $\nu^{\prime}$ be the corresponding measures. It is clear that $Z_{n}=\boldsymbol{X}_{n}-\boldsymbol{X}_{\| \prime}^{\prime}$. $Z$ $\boldsymbol{X}-\boldsymbol{X}^{\prime}, v_{n}=\mu_{n} * \tilde{\mu}_{n}$, and $v=\mu * \tilde{\mu}$ where $\boldsymbol{X}_{n}^{\prime}$ and $\boldsymbol{X}^{\prime}$ are independent, identical copies of $\boldsymbol{X}_{n}$ and $\boldsymbol{X}$ respectively, and $\tilde{\mu}_{n}(E)=\mu_{n}(-E)$. Let $(\Omega, P)$ be the corresponding probability space for $\left\{\tilde{\boldsymbol{\delta}}_{n}\right\}_{n-0}^{\infty}$. The following lemma is due to Garsia (1962): we include a proof here for completeness.

Lemma 3.3. For any $c>0$, there exists $C_{1}, C_{2}$ such that

$$
C_{1} S^{2}\left(c \rho^{n}\right) \leqslant P\left(\left|Z_{n}\right|<c \rho^{n}\right) \leqslant C_{2} S^{2}\left(c \rho^{n}\right)
$$

Proof. Without loss of generality we assume that $c=1$. Let $z_{n}=\sum_{k+0}^{n} \rho^{k} \tilde{s}_{k}, x_{i n}=\sum_{k}^{n}$ $\rho^{k} \varepsilon_{k}$ and $x_{n}^{\prime}=\sum_{k=0}^{n} \rho^{k} \varepsilon_{k}^{\prime}, \varepsilon_{k}, c_{k}^{\prime}=0$ or 1 , then

$$
\left\{z_{n}:\left|z_{n}\right|<\rho^{n}\right\}=\left\{x_{n}-x_{n}^{\prime}:\left|x_{n}-x_{n}^{\prime}\right|<\rho^{n}\right\} .
$$

Let $E_{i}=\left((i-1) \rho^{n}, i \rho^{n}\right]$, then

$$
\begin{aligned}
& \sum_{i} \mu_{n}\left(E_{i}\right)^{2}=\sum_{i} \int_{E_{i}}\left(\int \chi_{t}(x) \mathrm{d} \mu_{n}(x)\right) \mathrm{d} \mu_{n}\left(x^{\prime}\right) \\
& =\sum_{i} \int_{E_{i}}\left(\int \chi E_{i} \cdots y^{\prime}\left(x-x^{\prime}\right) \mathrm{d} \mu_{n}(x)\right) \mathrm{d} \mu_{n}\left(x^{\prime}\right) \\
& \leqslant \sum_{i} \int_{E_{i}}\left(\int \chi_{\left\{z_{n}:\left|z_{i j}\right|<\mu^{\prime \prime}\right\}}\left(x-x^{\prime}\right) \mathrm{d} \mu_{n}(x)\right) \mathrm{d} \mu_{n}\left(x^{\prime}\right) \\
& =\int_{-\infty}^{\infty}\left(\int \gamma_{\left\{z_{n}:|z|<\mu^{\prime \prime}\right\}}\left(x-x^{\prime}\right) \mathrm{d} \mu_{n}(x)\right) \mathrm{d} \mu \mu_{n}\left(x^{\prime}\right) \\
& =\mu_{n} * \tilde{\mu}_{n}\left\{z_{n}:\left|z_{n}\right|<\rho^{\prime \prime}\right\}=v_{n}\left\{z_{n}:\left|z_{n}\right|<\rho^{\prime \prime}\right\},
\end{aligned}
$$

and the first inequality follows. On the other hand, if $\left|z_{n}\right|<\rho^{n}$. then $z_{n}=x_{n} \cdots x_{n}^{\prime}$. For each such $x_{n} \in E_{k}, x_{n}^{\prime}$ can only be in $E_{k}$ or in the two adjacent intervals. Schwarz inequality then yields

$$
v_{n}\left\{z_{n}:\left|z_{n}\right|<\rho^{n}\right\} \leqslant \sum_{k} \mu_{n}\left(E_{k}\right)\left(\mu_{n}\left(E_{k-1}\right)+\mu_{n}\left(E_{k}\right)+\mu_{n}\left(E_{k+1}\right)\right) \leqslant 3 \sum_{k} \mu\left(E_{k}\right)^{2} .
$$

The lemma now follows from Proposition 3.2. 
Theorem 3.4. Let $\mu$ be an ICBM defined by $\frac{1}{2}<\rho<1$. Then for any $c>0$,

$$
\operatorname{dim}_{2}(\mu)=\lim _{n \rightarrow \infty} \frac{\ln P\left(\left|Z_{n}\right|<c \rho^{n}\right)}{n \ln \rho} .
$$

Furthermore, $\mu$ is absolutely continuous with $\mathrm{d} \mu / \mathrm{d} x \in L^{2}(\mathbb{R})$ if and only if

$$
\varlimsup_{n \rightarrow \infty} \frac{P\left(\left|\boldsymbol{Z}_{n}\right|<c \rho^{n}\right)}{\rho^{n}}<\infty .
$$

Proof. The first assertion is a consequence of Proposition 3.2 and Lemma 3.3. The second assertion was proved by Kahane and Salem (1958) using Fourier transformation. For our alternative proof, we make use of Lemmas 3.2 and 3.3 again to reduce the statement to: $\mu$ is absolutely continuous and $\mathrm{d} \mu / \mathrm{d} x \in L^{2}(\mathbb{R})$ if and only if

$$
\varlimsup_{h \rightarrow 0^{+}} \frac{1}{h^{2}} \int_{-\infty}^{\infty}|F(x+h)-F(x-h)|^{2} \mathrm{~d} x<\infty,
$$

where $F(x)=\mu(-\infty, x]$. This is a well-known theorem by Hardy and Littlewood (1928).

In the following we will use the above theorem to calculate the $L^{2}$-dimension for the special class of ICBM generated by the P.V. numbers. Let

$$
A_{n}=\left\{z_{n}=\sum_{k=0}^{n} \rho^{k} \tilde{\varepsilon}_{k}: \tilde{\varepsilon}_{k}=0, \pm 1, k=0, \ldots, n,\left|z_{n}\right|<\frac{\rho^{n+1}}{1-\rho}\right\} .
$$

Let $\beta=\rho^{-1}$, we rescale $A_{n}$ by multiplying $\beta^{n}$ by each element of $A_{n}$ and let

$$
B=\bigcup_{n=1}^{\infty}\left\{y_{n}=\sum_{k=0}^{n} \beta^{n-k} \tilde{\varepsilon}_{k}: \tilde{\varepsilon}_{k}=0, \pm 1, k=0, \ldots, n,\left|y_{n}\right|<\frac{1}{\beta-1}\right\} .
$$

Note that $\rho /(1-\rho)=1 /(\beta-1)$ is $c$ in Theorem 3.4, and it has the following special property:

Proposition 3.5. If $\left|y_{n}\right| \geqslant 1 /(\beta-1)$, then $\left|y_{n+1}\right| \geqslant 1 /(\beta-1)$.

Proof. The statement is a direct consequence of the following observation:

$$
\left|y_{n+1}\right|=\left|\beta y_{n}+\tilde{\varepsilon}_{n+1}\right| \geqslant \frac{\beta}{\beta-1}-1=\frac{1}{\beta-1} \text {. }
$$

Let $\boldsymbol{Y}_{n}=\sum_{k=0}^{n} \beta^{n-k} \tilde{\boldsymbol{\varepsilon}}_{k}$, then $\left\{\boldsymbol{Y}_{n}\right\}_{n=1}^{\infty}$ is a Markov chain. Proposition 3.5 says that once a path $\left\{y_{n}\right\}_{n=1}^{\infty}$ steps outside the barriers $\pm 1 /(\beta-1)$, then it will never return; $B$ is hence a set of inessential states of the Markov chain (i.e. for $y \in B$, there exists $y^{\prime} \notin B$ such that $y \rightarrow y^{\prime}$ but $y^{\prime} \nrightarrow y$ (Seneta, 1981, p.12). There may be finitely or infinitely many states in $B$.

Proposition 3.6. For $\frac{1}{2}<\rho<1$ and $\beta=\rho^{-1}$ a P.V. number, $B$ is a finite set. 
Proof. An elementary proof of this proposition is given in (Lau, 1993, Theorem 2.3). It also follows from an interesting estimation of Garsia [1962, Lemma 1.51]: Let $x>1$ be an algebraic integer, $x_{1}, \ldots, \alpha_{s}$ be its algebraic conjugates and let $\sigma$ denote the number of $\alpha_{i}$ such that $\left|\alpha_{i}\right|=1$. For any $n$th degree polynomial $L$ with integer coefficients $a_{i}$ and height $M:=\max \left\{\left|a_{i}\right|: i=1, \ldots, n\right\}$, if $L(\alpha) \neq 0$, then

$$
|L(x)| \geqslant \frac{\prod_{\left|x_{i}\right| \neq 1}|| \alpha_{i}|-1|}{(n+1)^{\sigma}\left(\prod_{\alpha_{,}>1}\left|\alpha_{i}\right|\right)^{n+1} M^{s}} .
$$

Now if $\beta$ is a P.V. number, then for $y_{n} \neq y_{m}$ in $B$ with $n \geqslant m$,

$$
y_{n}-y_{m}=\sum_{k=0}^{n} \beta^{n-k} \tilde{\varepsilon}_{k}-\sum_{k=0}^{m} \beta^{m-k} \tilde{\varepsilon}_{k}^{\prime}
$$

We use this to define a polynomial $L$ with coefficients $\tilde{\varepsilon}_{h}-\tilde{z}_{k}^{\prime}$ (letting $\varepsilon_{k}^{\prime}=0$ for $m<k \leqslant n)$. In this case $L$ has height at most 2. Also from the definition of P.V. number, the set $\left\{\beta_{i}:\left|\beta_{i}\right|>1\right\}$ is an empty set and $\sigma=0$. It follows that

$$
|L(\beta)| \geqslant \frac{\prod\left(1-\left|\beta_{i}\right|\right)}{2^{s}}:=C .
$$

I.e. if $y_{n} \neq y_{m}$, then $\left|y_{n}-y_{m}\right| \geqslant C$. Since all the elements of $B$ are bounded in between $\pm 1 /(\beta-1), B$ must be a finite set.

By using Propositions 3.5 and 3.6, we can define a substochastic matrix $Q$ of the Markov chain $\left\{\boldsymbol{Y}_{n}\right\}_{n=1}^{\infty}$ on the inessential states $B$. For each $y \in B$, we construct the corresponding row in $Q$ as follows: let $y^{\prime}=\beta y+\varepsilon, \tilde{\varepsilon}=0, \pm 1$; if $y^{\prime} \notin B$, we ignore the state; if $y^{\prime} \in B$ and $\tilde{\varepsilon}=1$ or -1 , we assign probability $\frac{1}{4}$ to the $y^{\prime}$; and if $y^{\prime} \in B$ and $\tilde{\xi}=0$, we assign $\frac{1}{2}$ instead.

As an example, let $\rho=(\sqrt{5}-1) / 2$. Then $\beta=\rho^{-1}=(\sqrt{5}+1) / 2$ is a P.V. number, it satisfies $\beta^{2}-\beta-1=0$. By using this it is easy to show that $B=\{0,1, \rho, \cdots 1,-, 1\}$ and the corresponding substochastic matrix is

$$
Q=\frac{1}{4}\left[\begin{array}{lllll}
2 & 1 & 0 & 1 & 0 \\
0 & 0 & 1 & 0 & 0 \\
1 & 2 & 0 & 0 & 0 \\
0 & 0 & 0 & 0 & 1 \\
1 & 0 & 0 & 2 & 0
\end{array}\right]
$$

Theorem 3.7. For $\frac{1}{2}<\rho<1$ such that $\beta=\rho^{-1}$ is a P.V. number, let $\mu$ be the corresponding ICBM. Let $Q$ be the substochastic matrix defined as above and let i be the maximal eigenvalue of $\boldsymbol{Q}$. Then the $L^{2}$-dimension of $\mu$ is $|\ln \lambda / \ln \rho|$. 
Proof. It follows from Seneta (1981, p.121) that

$$
P\left(\left|\boldsymbol{Z}_{n}\right|<\frac{\rho^{n+1}}{1-\rho}\right)=P\left(\left|\boldsymbol{Y}_{n}\right|<\frac{1}{\beta-1}\right)=\boldsymbol{v}_{0} \boldsymbol{Q}^{n} \mathbf{1},
$$

where $v_{0}$ is the row vector which equals 1 at state 0 , and 0 at the other states $y \in B$. Let $i$ be the positive maximal eigenvalue of $\boldsymbol{Q}$.

If $\boldsymbol{Q}$ is irreducible, then there exist a left eigenvector $\boldsymbol{u}_{f}$ and a right eigenvector $\boldsymbol{u}_{\mathrm{r}}$ of $Q$ corresponding to $\lambda$ such that each coordinate of these two eigenvectors is strictly positive. From this we see that for some positive constants $C_{1}, C_{2}$,

$$
C_{1} \boldsymbol{v}_{0} Q^{n} \boldsymbol{u}_{\mathrm{r}} \leqslant \boldsymbol{v}_{0} Q^{n} \mathbf{1} \leqslant C_{2} \boldsymbol{u}_{/} Q^{n} \mathbf{1}
$$

This implies that $\boldsymbol{v}_{0} Q^{n} \mathbf{1}$ is of order $\lambda^{n}$. For $x=|\ln \lambda / \ln \rho|$,

$$
0<\varliminf_{n \rightarrow \infty} \frac{P\left(\left|Z_{n}\right|<\frac{\rho^{n+1}}{1-\rho}\right)}{\rho^{\alpha n}} \leqslant \varlimsup_{n \rightarrow \infty} \frac{P\left(\left|Z_{n}\right|<\frac{\rho^{n+1}}{1-\rho}\right)}{\rho^{\alpha n}}<\infty,
$$

and hence

$$
\operatorname{dim}_{2}(\mu)=\lim _{n \rightarrow \infty} \frac{\ln P\left(\left|Z_{n}\right|<\frac{\rho^{n+1}}{1-\rho}\right)}{n \ln \rho}=\alpha .
$$

If $Q$ is reducible, for simplicity, we assume $Q$ can be reduced into two components

$$
Q=\left[\begin{array}{cc}
Q_{1} & R \\
0 & Q_{2}
\end{array}\right]
$$

where $Q_{1}$ and $Q_{2}$ are irreducible, with the 0 th row corresponding to state 0 , which reaches all the states. It is easy to prove (3.2) from this, and (3.3) follows.

In the above example of $\rho=(\sqrt{5}-1) / 2$, we can use symmetry to identify the states -1 and $-\rho$ with 1 and $\rho$. The corresponding matrix $Q^{\prime}$ on $\{0,1, \rho\}$ is given by

$$
Q^{\prime}=\frac{1}{4}\left[\begin{array}{lll}
2 & 2 & 0 \\
0 & 0 & 1 \\
1 & 2 & 0
\end{array}\right]
$$

( $Q^{\prime}$ is obtained by cutting the last two rows and flipping the last two columns for states -1 and $-\rho$ to the columns for 1 and $\rho$.) These two matrices have the same maximal eigenvalue which is the larger positive root of the polynomial

$$
(4 \lambda)^{3}-2(4 \lambda)^{2}-2(4 \lambda)+2=0 .
$$

By Theorem 3.7, $\operatorname{dim}_{2}(\mu)=|\ln \lambda / \ln \rho|$. The reduction to the smaller matrix also works for the other P.V. numbers (Lau, 1993). Table 1 gives a list of P.V. numbers for which $\operatorname{dim}_{2}(\mu)$ has been calculated, using Theorem 3.7. The minimal polynomial is the defining equation for $\beta=\rho^{-1}$. (The fourth row is the case of the golden number.) 
Table 1

\begin{tabular}{|c|c|c|c|c|}
\hline Min. Polynomial & $\rho$ & Size of $Q^{\prime}$ & $4 \lambda$ & $\operatorname{dim}_{2}\left(\mu_{1}\right)$ \\
\hline$x^{5}-x^{4} \ldots-1=0$ & 0.5086604 & 6 & 2.0573712 & 0.9835654 \\
\hline$x^{4}-\cdots x^{3} \cdots-1=0$ & 0.5187901 & 5 & 2.1118009 & 0.9733295 \\
\hline$x^{3}-x^{2} \cdots x-1=0$ & 0.5436890 & 4 & 2.2226941 & 0.9642200 \\
\hline$x^{2} \quad x-1=0$ & 0.6180334 & 3 & 2.4811943 & 0.0923094 \\
\hline$x^{3}-x^{2} \ldots 1=0$ & 0.6823275 & 25 & 2.7302333 & 0.999116. \\
\hline$x^{4}-x^{3}-1-0$ & 0.7244919 & 627 & 2.8979776 & 0.9099895 \\
\hline$x^{3}-x-1=0$ & 0.7548776 & 90 & 3.0195190 & 0.999090 \\
\hline
\end{tabular}

\section{The golden number}

In this section we will give a formula of the $L^{p}$-dimension of the ICBM corresponding to $\rho=(\sqrt{5}-1) / 2$. The technique is to use the self-similar identity (1.1) to obtain a functional equation: the renewal equation (Feller, 1971). We will first use the simpie case $0<\rho<\frac{1}{2}$ to demonstrate the idea. Let $S_{1}, S_{2}$ be the similitudes defined as in (1.2) with $0<p<\frac{1}{2}$ and let

$$
\Phi^{(x)}(h)=\frac{1}{h^{1+x}} \int \mu\left(B_{h}(x)\right)^{p} \mathrm{~d} x .
$$

By using

$$
\mu=\frac{1}{2}\left(\mu \circ S_{1}^{-1}+\mu \circ S_{2}^{-1}\right) \text { and } S_{1}[0,1] \cap S_{2}[0,1]=\emptyset .
$$

we have for $h$ sufficiently small, $B_{h}\left(S_{1}^{-1}(x)\right) \cap B_{h}\left(S_{2}^{-1}(x)\right)=\emptyset$ and

$$
\begin{aligned}
\Phi^{(x)}(h) & =\frac{1}{2^{p} h^{1+x}} \int\left(\mu\left(B_{h}\left(\rho^{-1} x\right)\right)+\mu\left(B_{h}\left(\rho^{-1}(x-(1-\rho))\right)\right)^{p} \mathrm{~d} x\right. \\
& =\frac{1}{2^{p} h^{1+x}}\left(\int \mu\left(B_{h}\left(\rho^{-1} x\right)\right)^{p} \mathrm{~d} x+\int \mu\left(B_{h}\left(\rho^{-1}(x-(1-\rho))\right)\right)^{\prime \prime} \mathrm{d} x\right) \\
& =\frac{\rho}{2^{p-1} h^{1+x}} \int \mu\left(B_{h ; \rho}(x)\right)^{p} \mathrm{~d} x \quad \text { (by a change of variables) } \\
& =\frac{\rho^{x}}{2^{p-1}} \Phi^{(x)}\left(\frac{h}{\rho}\right) .
\end{aligned}
$$

If we choose $x=|(p-1) \ln 2 / \ln \rho|$, then the above multiplicative periodicity implies that

$$
0<\varliminf_{h \rightarrow 0^{+}} \Phi^{(x)}(h) \leqslant \varlimsup_{h \rightarrow 0^{-}} \Phi^{(x)}(h)<\infty,
$$

and by (2.1) and Lemma 2.2 , the $l^{p}$-dimension of $\mu$ is $|\ln 2 / \ln \rho|$ for $p \geqslant 1$. 
When $\rho=(\sqrt{5}-1) / 2$, the maps $S_{1}, S_{2}$ do not satisfy the disjointness condition in (4.1) and the second equality in the above calculation will not hold. To overcome this, we make use of a device of Strichartz et al. (to appear): Let

$$
\begin{aligned}
& T_{0} x=S_{1} S_{1} x=\rho^{2} x, \\
& T_{1} x=S_{2} S_{1} S_{1} x=S_{1} S_{2} S_{2} x=\rho^{3} x+\rho^{2}, \\
& T_{2} x=S_{2} S_{2} x=\rho^{2} x+\left(1-\rho^{2}\right) .
\end{aligned}
$$

Note that $T_{i}(0,1), i=0,1,2$ are the three intervals $\left[0, \rho^{2}\right],\left[\rho^{2}, \rho\right]$ and $[\rho, 1]$, and that $(0,1)$ is the disjoint union of these three intervals. The disjointness condition in (4.1) is satisfied (excluding the two end points). On the other hand, the self-similar identity in (4.1) is reduced to the following "second order" self-similar identities defined by the $T_{i}$ 's: For $A \subseteq[0,1]$,

$$
\left[\begin{array}{l}
\mu\left(T_{0} T_{i} A\right) \\
\mu\left(T_{1} T_{i} A\right) \\
\mu\left(T_{2} T_{i} A\right)
\end{array}\right]=P_{i}\left[\begin{array}{l}
\mu\left(T_{0} A\right) \\
\mu\left(T_{1} A\right) \\
\mu\left(T_{2} A\right)
\end{array}\right]
$$

where

$$
P_{0}=\left[\begin{array}{ccc}
\frac{1}{4} & 0 & 0 \\
\frac{1}{8} & \frac{1}{4} & 0 \\
0 & \frac{1}{2} & 0
\end{array}\right], \quad P_{1}=\left[\begin{array}{ccc}
0 & \frac{1}{4} & 0 \\
0 & \frac{1}{4} & 0 \\
0 & \frac{1}{4} & 0
\end{array}\right] \text { and } P_{2}=\left[\begin{array}{ccc}
0 & \frac{1}{2} & 0 \\
0 & \frac{1}{4} & \frac{1}{8} \\
0 & 0 & \frac{1}{4}
\end{array}\right]
$$

It follows from (4.2) that for $A \subseteq[0,1]$ and for $J=\left(j_{1}, \ldots, j_{k}\right)$ with $j_{i}=0$ or 2 ,

$$
\mu\left(T_{1} T_{J} T_{1} A\right)=c_{J} \mu\left(T_{1} A\right) \quad \text { where } c_{J}=\frac{1}{4}[0,1,0] P_{J}\left[\begin{array}{l}
1 \\
1 \\
1
\end{array}\right]
$$

where $T_{J}=T_{j_{1}} \cdots T_{j_{k}}$ and likewise for $P_{J}$. To calculate the $L^{p}$-dimension of $\mu$, we use (4.2) to produce a functional equation for $\int_{0}^{1} \mu\left(B_{h}(x)\right)^{p} \mathrm{~d} x$ as in the proof of the first paragraph. Observe that

$$
\begin{aligned}
& \int_{0}^{1} \mu\left(B_{h}(x)\right)^{p} \mathrm{~d} x=\left(\int_{T_{0}[0,1]}+\int_{T_{1}[0,1]}+\int_{T_{[}[0,1]}\right) \mu\left(B_{h}(x)\right)^{p} \mathrm{~d} x \\
& =\rho^{2} \int_{0}^{1} \mu\left(B_{h}\left(T_{0} x\right)\right)^{p} \mathrm{~d} x+\rho^{3} \int_{0}^{1} \mu\left(B_{h}\left(T_{1} x\right)\right)^{p} \mathrm{~d} x+\rho^{2} \int_{0}^{1} \mu\left(B_{h}\left(T_{2} x\right)\right)^{p} \mathrm{~d} x .
\end{aligned}
$$


Let

$$
\Phi_{i}^{(\alpha)}(h)=\frac{1}{h^{1+\alpha}} \int_{0}^{1} \mu\left(B_{h}\left(T_{i} x\right)\right)^{p} \mathrm{~d} x, \quad i=0,1,2 .
$$

Then $\Phi_{0}^{(x)}(h)=\Phi_{2}^{(x)}(h)$ and $\Phi_{1}^{(x)}(h)$ dominates the other two (see the second picture at the end of Section 5, the three $\Phi_{i}^{(x)}(h)$ 's are determined by $\mu$ on the three intervals $\left[0, \rho^{2}\right],\left[\rho^{2} . \rho\right]$ and $[\rho, 1]$ respectively). By repeating the above argument of splitting the interval $[0,1]$ of $\Phi_{1}^{(x)}$ into three pieces as in (4.4), applying (4.2), (4.3) and using the change of variables, we have

$$
\Phi_{1}^{(x)}(h)=\sum_{k=0}^{\infty}\left(\sum_{|J|=k} c_{J}^{p}\right) \rho^{-(2 k+3) \alpha} \Phi_{1}^{(\alpha)}\left(\frac{h}{\rho^{(2 k-3)}}\right)+\mathrm{o}\left(h^{\delta}\right) .
$$

To solve $\Phi_{1}^{(x)}$, we use $x=-\ln h$ to change the equation into the following convolution equation (renewal equation):

$$
f(x)=\int_{0}^{x} f(x-y) \mathrm{d} \sigma(y)+S(x), \quad x \geqslant 0
$$

where $f, \sigma$, and $S$ are defined in the obvious way; $S(x)$ is a continuous integrable function with $\lim _{x \rightarrow x} S(x)=0$. In order to have a nontrivial bounded solution, $\sigma$ must be a probability measure (Feller, 1971). This implies that if we take $(p, x)$ such that

$$
F(p, x):=\sum_{k=0}^{\infty}\left(\sum_{|J|=k} c_{J}^{p}\right) \rho^{-(2 k+3) x}=1,
$$

we have

$$
0<\varliminf_{h \rightarrow()^{-}} \Phi_{1}^{(x)}(h) \leqslant \varlimsup_{h \rightarrow 0^{+}} \Phi_{1}^{(x)}(h)<\infty .
$$

From this we can apply (2.1) and Lemma 2.2 to obtain

Theorem 4.1. For $\rho=(\sqrt{5}-1) / 2$. Suppose $p>0$, then there exists a unique $x$ suth that $F(p, x)=1$ and $\operatorname{dim}_{p}=\alpha /(p-1)$.

The derivation of Eq. (4.5) and the proof of the theorem involves some technical estimations, the details are given by Lau and Ngai. By using the theorem we can prove the following result which is useful for computation.

Theorem 4.2. If $p$ is a nonnegative integer, then $F(p, x)=1$ can be reduced to a polynomial equation, and the solution $x$ corresponds to the largest positive root of the polvnomial.

For example if $p=2$, then the polynomial equation is $\left(4 \rho^{x}\right)^{3}-2\left(4 \rho^{x}\right)^{2}-2\left(4 \rho^{x}\right)+$ $2=0$ (the same as in $(3.4)$ ) and $\operatorname{dim}_{2}(\mu) \approx 0.99240$. If $p=3$, then corresponding equation is $\left(8 \rho^{x}\right)^{3}-2\left(8 \rho^{x}\right)^{2}-4\left(8 \rho^{x}\right)+2=0$. and $\operatorname{dim}_{3}(\mu) \approx 0.98971$. 
In Section 2 we mentioned that heuristically the entropy dimension is the limit of the $L^{p}$-dimension as $p \rightarrow 1$ using the L'Hôspital's rule. In the present case the statement can be justified and we have

Proposition 4.3. Let $\mu$ be defined as above, then

$$
\operatorname{dim}_{1}(\mu)=\frac{1}{9 \ln \rho_{k}} \sum_{k=0}^{\infty}\left(\sum_{|J|=k} c_{J} \ln c_{J}\right) .
$$

There are different formulae for the entropy dimension (Garsia, 1963; Alexander and Yorke, 1984; Alexander and Zagier, 1991; Ledrappier and Porzio). Using the above formula we can calculate that $\operatorname{dim}_{1}(\mu) \approx 0.99571$ which is compatible with the previous result. Also $\mathrm{Hu}$ (to appear) used an algebraic method and showed that $\operatorname{dim}_{\infty}(\mu)=-(1 / 2)-\ln 2 / \ln \rho \approx 0.9404$. It is seen that the spectrum of $L^{p}$-dimension lies in a very narrow band close to 1 .

\section{Remarks and open problems}

The technique to reduce the overlapping case to a nonoverlapping case in the last section is quite restrictive. Besides the golden number, another P.V. number has been found to have the same property $\left(\rho^{-1}\right.$ satisfies $\left.x^{3}-2 x^{2}+x-1=0\right)$, but most of them do not.

Question 1. Might one obtain an explicit formula of $\operatorname{dim}_{p}(\mu)$ as in Theorem 4.1 where $\mu$ is generated by a P.V. number?

In Section 3, the calculation of the $L^{2}$-dimension depends on Proposition 3.6 that the states in $B$ is a finite set.

Question 2. Does this property characterize the P.V. numbers?

A positive answer would give a new characterization of the P.V. number, however a negative answer would be even more interesting because it would give a new singular ICBM.

Finally we return to the absolutely continuous case. Although Solomyak (1995) proved that for almost all $\frac{1}{2}<\rho<1$, the corresponding ICBM is absolutely continuous, his proof does not offer any number explicitly. A very natural and important question is

Question 3. Is the ICBM corresponding to $\frac{1}{2}<\rho<1$ where $\rho$ is a rational number absolutely continuous?

In the following we draw the graphs (Figs. 1 and 2) of the approximate densities, $\mu(x-h, x+h) / 2 h$ for $h$ small, of the ICBMs corresponding to $\rho=\frac{1}{3},(\sqrt{5}-1) / 2, \frac{2}{3}$ and $\frac{3}{4}$. It is seen that in the case $\rho=\frac{1}{3}$, the measure is very singular and is concentrated on the Cantor set in a uniform manner; this can be used to explain that the $L^{p}$-dimension 

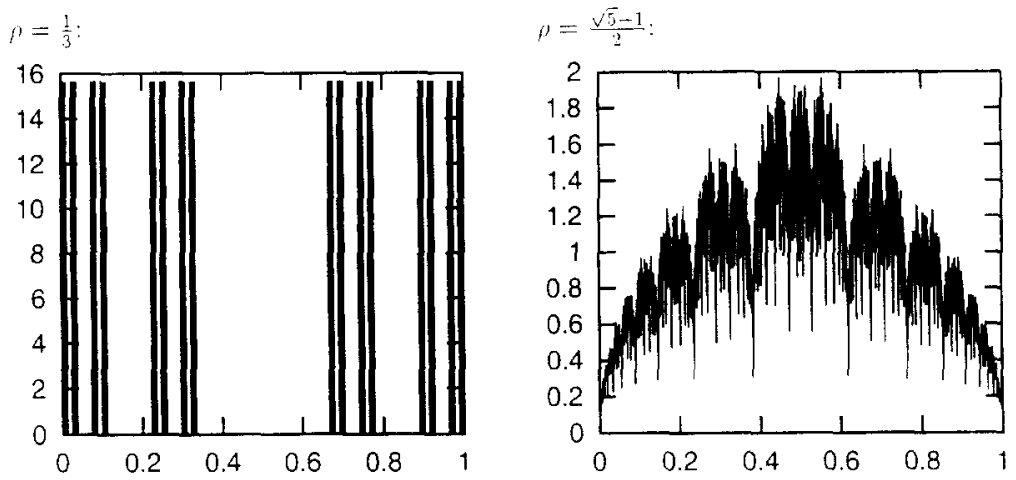

Fig. 1.
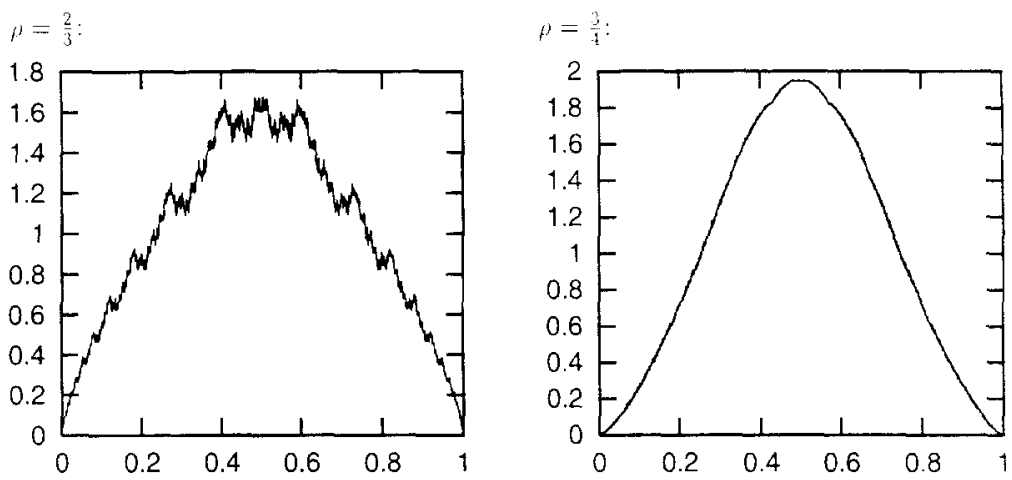

Fig. 2.

( $=|\ln 2 / \ln \rho|$ ) is independent of $p$ (see the first paragraph of Section 4). For the case $\rho=(\sqrt{5}-1) / 2, \mu$ is also singular and is concentrated on a dense subset of $[0,1]$, which is composed of sets of different local dimension; it is a multifractal measure (Lau and Ngai, to appear). For the cases $\rho=\frac{2}{3}$ and $\rho=\frac{3}{4}$, the pictures suggested that both measures are absolutely continuous, as is asked in Question 3.

\section{Acknowledgements}

The authors thank the referees for many valuable suggestions to improve the paper, This research was partially supported by the REU grant from Cornell University and a research grant from the National Science Council of the Republic of China. Both authors would like to thank Professor R. Strichartz for his arrangement to visit Cornell University and for many valuable discussions and suggestions. The first author thanks Professor W.J. Huang, C.K. Law and N.C. Wong for their hospitality and help while he was visiting the Department of Applied Mathematics, National Sun Yat-Sen 
University. Finally, the first author would like to express his deepest gratitude to Professor C.R. Rao from whom he learned the application of functional equations in probability and statistics.

\section{References}

Alexander, J., Yorke, J., 1984. Fat baker's transformations. Ergodic Theory Dynamical Systems 4, 1-23.

Alexander, J., Zagier, D., 1991. The entropy of a certain infinitely convolved Bernoulli measure. J. London Math. Soc. 44, 121-134.

Bovier, A., Bernoulli convolutions, dynamical systems and automata, preprint.

Erdös, P., 1939. On a family of symmetric Bernoulli convolutions. Amer. J. Math. 61, 974-976.

Erdös, P., 1940. On the smoothness properties of a family of symmetric Bernoulli convolutions. Amer. J. Math. 62, 180-186.

Falconer, K., 1990. Fractal Geometry - Mathematical Foundation and Applications. Wiley, New York.

Feller, W., 1971. An Introduction to Probability Theory and its Applications, vol II, 2nd ed. Wiley, New York.

Garsia, A., 1962. Arithmetic properties of Bernoulli's convolutions. Trans. Amer. Math. Soc. 102, 409-432.

Garsia, A., 1963. Entropy and singularity of infinite convolutions. Pacific J. Math. 13, $1159-1169$.

Hardy, G., Littlewood, J., 1928. Some property of fractional integrals. Math. Z. 27, 565-606.

Hu, T.Y., The local dimension of the Bernoulli convolution associated with the golden number. Trans. Amer. Math. Soc., to appear.

Hutchinson, J., 1981. Fractal and self-similarity. Indiana Univ. Math. J. 30, 713-747.

Jessen, B., Wintner, A., 1935. Distribution functions and Riemann zeta function. Trans. Amer. Math. Soc. $38,48-88$.

Kahane, J.P., Salem, R., 1958. Sur la convolution d'une infinit de distributions de Bernoulli 6, 193-202.

Lalley, S., Random series in powers of algebraic integers: Hausforff dimension of the limit distribution, preprint.

Lau, K.S., 1992. Fractal measures and mean p-variations. J. Funct. Anal. 108, 427-457.

Lau, K.S., 1993. Dimension of a family of singular Bernoulli convolutions. J. Funct. Anal. 116, $335-358$.

Lau, K.S., Ngai, S.M., Multifractal measure and a weak separation condition. Adv. in Math., to appear.

Lau, K.S., Ngai, S.M., The $L^{q}$-dimension of the Bernoulli convolution associated with the golden number, preprint.

Ledrappier, F., Porzio, A., A dimension formula for Bernoulli convolution, preprint.

Przytycki, F., Urbanski, 1989. On the Hausdorff dimension of some fractal sets. Studia Math. 93, $155-158$.

Rényi, A., 1957. Dimension, entropy and information. In: Trans. 2nd Prague Conf. Inform. theory Statist. Decision. Funct., Random Processes, pp. 545-556.

Salem, R., 1962. Algebraic Numbers and Fourier Transformations, Heath Math. Monographs. Boston.

Seneta, E., 1981. Non-negative Matrices and Markov Chains, 2nd ed. Springer Series in Statistics, New York.

Solomyak, B., 1995. On the random series $\Sigma \pm i^{n}$ (an Erdös problem). Annal of Math. 142, $611-625$.

Strichartz, R., Taylor, A., Zhang, T., Densities of self-similar measures. Experimental Math., to appear.

Wiener, N., Wintner, A., 1939. On singular distributions. J. Math. Phys. 17, 233-346. 